\title{
A selection of relevant issues in applied stochastic frontier analysis
}

\author{
Antonio Alvarez $^{1 \star} \cdot$ Carlos Arias $^{2}$ \\ ${ }^{1}$ Oviedo Efficiency Group, Department of Economics, University of Oviedo, Spain \\ ${ }^{2}$ Oviedo Efficiency Group, Department of Economics, University of León, Spain
}

\begin{abstract}
This paper provides a concise review of a broad set of concepts, models and estimation issues in the field of stochastic frontier analysis. The goal is to provide practitioners with a quick guide to some key ideas. The review is restricted to single-output models estimated by econometric methods. Among the conceptual issues are the interpretation and the paths to reduce inefficiency. The modelling and estimation parts deal with topics such as explaining inefficiency, time-varying inefficiency and firm heterogeneity.
\end{abstract}

Keywords: stochastic frontiers, technical inefficiency, management, time-varying inefficiency, latent class models

JEL Classification Codes: C51, D21, D24

\section{Introduction}

Stochastic frontier analysis dates back to the seminal papers of Aigner, Lovell and Schmidt (1977) and Meeusen and van den Broeck (1977). Their original model has been extended in many ways to account for some important topics such as allowing inefficiency to vary over time, explaining inefficiency and controlling for other types of unobserved heterogeneity. The estimating techniques have also become more sophisticated and although the original ML estimator is still standard, other techniques (Bayesian, semi-parametric...) are increasingly common nowadays.

Therefore, the stochastic frontier literature has grown too large to try to tackle it in a comprehensive way in this short note. Instead, we have chosen to focus on three broad areas: concept, modelling and estimation issues. Our objective is to highlight some technical and conceptual issues that can be of use for practitioners. In choosing the issues we have made a somewhat inevitably personal selection based on topics which we and our closest collaborators have been most interested in and dedicated most time over the last two decades.

\footnotetext{
* Corresponding author. E-mail: alvarez@uniovi.es.

Citation: Alvarez, A. and Arias, C. (2014) A selection of relevant issues in applied stochastic frontier analysis, Economics and Business Letters, 3(1), 3-11.
} 


\section{Conceptual issues}

In what follows we will restrict ourselves to the case of a single output primal technology. In this context, the typical stochastic production frontier model can be written as:

$$
y_{i}=\alpha+\beta x_{i}+v_{i}-u_{i}
$$

where subscript $i$ refers to a production unit (firms, countries,...), $y$ is (log of) output, $x$ are inputs, $v$ is statistical noise and $u$ is a nonnegative random term that accounts for inefficiency.

The identification of the two random terms is based on different distributional assumptions for $u$ and $v$ plus the assumption that $u, v$ and $x$ are mutually independent. Note that this implies that input choices are not influenced by the level of technical efficiency.

In (1) an output-oriented index of technical efficiency can be computed as $\exp \left(-u_{i}\right)$. However, it is possible to choose several alternative directions to go to the frontier. Associated with each direction there is a different measure of technical efficiency (e.g., output-oriented, input-oriented, hyperbolic, etc.). At this point, it is important to note that theoretical analysis of technical efficiency is always carried out under the assumption that the technology is known. However, in empirical work the technology and the level of technical efficiency cannot be estimated independently. That is, it is necessary to choose an efficiency index (and therefore a model) prior to estimating the production technology.

The choice between an output-oriented and an input-oriented model is equivalent to a choice of additive versus interactive individual effects (the unobservable individual effect being the inefficiency term). In a primal setting, the output-oriented model can be cast as a model with an additive effect while the input-oriented model can be seen as a model with an interactive effect. The second model is harder to estimate and has therefore been used very rarely. Alvarez, Arias and Kumbhakar (2003) study the effects of direction choice not only on efficiency rankings but also on the estimated production technology. The results show that there are important differences in the estimated technological characteristics depending on the model selected. Orea, Roibas and Wall (2004) compared different specifications of the efficiency index and used the Vuong test to select the best model.

\subsection{The difficult task of interpreting (in)efficiency}

What is inefficiency? This question has a simple answer: inefficiency is distance from the frontier (given inputs). A more subtle question is what is the interpretation of inefficiency? From an empirical perspective, inefficiency is part of the error term and will, therefore, contain (part of) all omitted relevant variables. At this point we will discuss the issue of interpretation assuming that the model is correctly specified.

It is common (and reasonable) to interpret inefficiency as the result of inadequate management practices. In fact, this is the stand taken by Farrell (1957) in his classic paper. However, equating inefficiency with poor management does not imply that the role of management in production is being correctly modelled. Modelling management is problematic because it is unobservable and for this reason it has been omitted from many production models. This omission can lead to biased estimates of the remaining parameters of the production function. Economists have coined the term 'management bias' to refer to this problem and two remedies have been proposed in the literature. Following Mundlak (1961), some authors have used covariance analysis or similar tools to control for the effect of time invariant management, while other studies have used 'proxies' for management. For example, Alvarez and Arias (2003) develop a cost model where management is considered a fixed input. The model shows how increasing output with a fixed level of managerial ability can lead to an increase in observed economic inefficiency. This is important because in many instances economic policies aim to increase firm output without a careful analysis of the role 
played by managerial ability. Since management is unobserved, in the empirical exercise with dairy farms Alvarez and Arias (2003) use technical efficiency (estimated in a previous step) as a proxy for managerial ability and find that increasing farm size while holding managerial ability constant can reduce the extent of economies of size.

The analysis of covariance treats management as an additive unknown effect. However, the existence of interactions between management and input levels is an intriguing possibility. Alvarez, Arias and Greene (2005) explore this case by including fixed managerial ability as an unobservable input in a translog production function and explore its relationship with technical efficiency. An interesting implication of this model is that management is different from technical efficiency. In fact, the interaction between management and conventional inputs implies that technical efficiency depends not only on management but also on input use. As a result, fixed management can lead to time-varying technical efficiency if input use changes over time. This is an important insight not considered in models that impose timeinvariant efficiency based in the assumption of fixed management.

Fisheries provides a good example of a sector where management (skipper skill is the common term in this literature) is assumed to be an important factor in order to explain differences in catches across vessels. This is known as the "the good captain hypothesis", and has been tested in the framework of stochastic frontier models by assuming that management is modeled in the technical inefficiency term. The pervasive result was that skill is much more important than luck in explaining fish catches. However, Alvarez and Schmidt (2006) claim that this finding could be due to the aggregation of data over time (i.e. having daily catches but aggregating them at the monthly or yearly level). Using daily data of artisan fleet they find that luck is more important than technical efficiency in explaining catches and argue this can be explained by the fact that over longer periods of time, skill persists while luck averages out.

In summary, while interpreting the inefficiency measure as an indicator of mismanagement may seem reasonable, researchers are cautioned to pay attention to the implications of all modelling details.

\subsection{The even more difficult task of reducing inefficiency}

Technical inefficiency reflects the failure of some firms to obtain the maximum feasible output given the amount of inputs used. Its measurement is crucial to quantify the importance of poor performance in a productive activity. Unfortunately, measurement is not enough. In order to improve technical efficiency, firms should be able to identify the sources of underperformance and the alternatives available to make better use of their resources. Therefore, the question arises as to how a firm can become efficient in practice.

Current measures of technical efficiency are not that helpful when it comes to improving efficiency. The implicit assumption is that inefficient firms should behave as those on the best practice frontier. But behavior has two components: 'how much' the firms are doing and 'how' they do it. Efficiency measures only inform in terms of 'how much'. The frontier determines the input reduction an inefficient firm could achieve. However, if this inefficient firm reduces its input use but continues acting in the same manner as before the reduction, we would observe a firm that produces less output but is still inefficient.

Obviously, the solution for the inefficient firm is to find out what it is doing wrong and then correct its mistakes. The challenge is how to do this in practice. A reasonable strategy would be for the inefficient firm to visit some of the efficient firms to observe how they do things. This benchmarking procedure is common in farm management programs. A nontrivial question here is how to choose which of the efficient firms it should visit.

Gonzalez and Alvarez (2001) provide an operative way to find the closest reference firm in the efficient subset of the isoquant. The comparison group in the efficient subset will be 
composed of efficient firms that share the largest number of similarities in input endowments and which are therefore easier for the inefficient firm to imitate. The idea is that the inefficient firm may learn more from trying to emulate these firms than by looking at other efficient firms. To make this idea operative, they introduce the concept of input-specific contraction as a modified version of the single-factor efficiency measure used by Kopp (1981) ${ }^{1}$. Papers that use single-factor efficiency (e.g, Reinhard, Lovell and Thijssen, 1999) assume that any single input contraction is feasible. In turn, Gonzalez and Alvarez (2001) try to make sure that the contraction is realistic by using a close peer as a benchmark.

\section{Modelling issues}

In this section, we discuss three broad modelling issues. The first is the choice between modelling inefficiency as a random disturbance versus as a parameter. This discussion has a methodological flavour although with profound economic implications. Conversely, in the last two topics, interesting economic issues (time-varying efficiency and the causes of inefficiency) lead to a wealth of modelling proposals.

\subsection{Inefficiency as a random term versus inefficiency as a parameter}

In the stochastic frontier in (1) inefficiency is specified as a one-sided random disturbance. In fact, in a cross section the best hope is to estimate the parameters that characterize the distribution of inefficiency. However, with panel data it is possible to estimate individual efficiencies as parameters.

With panel data and assuming time-invariant inefficiency, model (1) can be rewritten as:

$$
y_{i t}=\alpha+\beta x_{i t}+v_{i t}-u_{i}=\alpha_{i}+\beta x_{i t}+v_{i t}
$$

where the parameters $\alpha_{i}=\alpha-u_{i}$ are individual fixed effects that can be estimated using the within estimator and the inefficiency term $u_{i}$ can be recovered afterwards. This is the approach followed by Schmidt and Sickles (1984) in a widely-cited paper that clarifies a whole set of issues on the estimation of time-invariant technical inefficiency with panel data.

The treatment of inefficiency as a parameter deals with two relevant issues. First, model (2) can be estimated without assuming a statistical distribution for inefficiency. Second, and in our view more important, we can avoid a subtle but essential assumption required for the estimation of a stochastic frontier, namely the absence of correlation between inefficiency and inputs. Such correlation will produce biased estimates of the parameters of the frontier. Additionally, the correlation between inputs and inefficiency can be a core issue in production models. For example, the analysis of the relationship between technical efficiency and size in Alvarez and Arias (2004) relies on the existence of a positive correlation between input demands and the level of technical efficiency.

\subsection{The evolution of inefficiency}

This is one of the most common objectives of efficiency studies. Researchers are interested in the evolution of firm efficiency over time. A common setting to study this issue is the following:

\footnotetext{
${ }^{1}$ These papers use non-parametric methods while the models reviewed in the present survey are parametric. Our intention is to underline a relevant conceptual issue abstracting from the methodology used in the papers.
} 


$$
y_{i t}=\alpha+\beta x_{i t}+v_{i t}-u_{i t}
$$

This is the equivalent of equation (1) for panel data, which implies the underlying assumption for the inefficiency term of $u_{i t} \sim N^{+}\left(\mu, \sigma_{\mathrm{u}}{ }^{2}\right)$. Therefore, inefficiency is assumed to come from a distribution that has both a mean and a variance which are constant over time. Obviously, this assumption precludes any temporal variation of efficiency besides random movements around a constant mean.

The key aspect to correctly modelling the evolution of inefficiency over time is to be able to define $\partial E\left(u_{i t}\right) / \partial t$ parametrically. Several papers have specified $u_{i t}$ as a parametric function of time. While the first contribution was due to Kumbhakar (1990), the most widely-used specification is the one by Battese and Coelli (1992), where $u_{i t}(t)=u_{i} \exp (\delta(T-t))$. Alternatively, some authors have chosen to model time-varying inefficiency as a parameter, i.e., $\alpha_{i t}=\alpha-u_{i t}$. Cornwell, Schmidt and Sickles (1990) proposed $\alpha_{i t}=\alpha_{i 0}+\alpha_{i 1} t+\alpha_{i 2} t^{2}$, while Lee and Schmidt (1993) proposed $\alpha_{i t}=\theta_{t} \alpha_{i}$. Ahn, Lee and Schmidt (2007) extend the model in Lee and Schmidt (1993) by including several time shocks common to all firms that multiply firmspecific effects. The result is a model with firm-specific time patterns of efficiency variation able to nest most of previous models.

What is the difference between these specifications? The key again is in $\partial E\left(u_{i t}\right) / \partial t$. The models by Cornwell, Schmidt and Sickles (1990) and Ahn, Lee and Schmidt (2007) allow for a firm-specific time pattern while the others assume a common time pattern for all firms. In other words, $\partial E\left(u_{i t}\right) / \partial t$ is different for each firm in this model while it is constant across firms in all other models considered above, implying that the temporal pattern of inefficiency is the same for all firms.

\subsection{Explaining inefficiency}

There are some papers in which efficiency is estimated in a first step and then the relationship between the level of efficiency and several explanatory variables $(z)$ is analyzed in a second step. This approach is unsatisfactory since excluding the explanatory variables of efficiency in the first stage will most likely yield biased estimates of the technological parameters Wang and Schmidt (2002) provide extensive Monte Carlo evidence that the size of this bias is important but also that the estimated efficiency levels are spuriously underdispersed (even if there is no correlation between the inputs and the $z^{\prime} \mathrm{s}$ ), causing the second-step regression to understate the effect of the $z$ 's on efficiency.

A rigorous approach to the problem consists of including the potential causes of inefficiency as explanatory variables of the distribution of the inefficiency term. The first papers that attempted to do so were Kumbhakar, Ghosh and McGuckin (1991), Reifschneider and Stevenson (1991) and Huang and Liu (1994). Their approach consists of making the mean or the variance of the pre-truncated distribution of the inefficiency term depend on a set of exogenous variables $(z)^{2}$. Battese and Coelli (1995) extended this approach to accommodate panel data, in the following way:

$$
u_{i t} \sim N^{+}\left(\mu_{i t}, \sigma_{u}^{2}\right), u_{i t} \geq 0, \mu_{i t}=\delta z_{i t}
$$

where $z_{i t}$ are variables that are assumed to affect inefficiency (e.g. size, time, form of ownership, managerial characteristics, etc.).

To analyze the effect of $z_{i t}$ we need to compute the marginal effect of $z_{i t}$ on the unconditional expectation of inefficiency, that is, $\partial E\left(u_{i t}\right) / \partial z$. However, in model (4) this expression is quite cumbersome (see Wang, 2002).

\footnotetext{
2 Alvarez et al. (2006) consider a model where both the mean and the variance of the distribution of $u$ are a function of $z$. They do so by 'scaling' the basic random variable $u$.
} 
An important feature of the Battese and Coelli (1995) model, which is shared by other models, is that it assumes independence over time of the inefficiency term. That is, a firm observed in two periods is treated as two different firms. This assumption does not allow us to estimate the inefficiency level consistently since its variance does not vanish as the sample size increases.

More recent models try to overcome these two problems. For example, Orea and Kumbhakar (2004) proposed the following specification:

$$
u_{i t}=g\left(z_{i t}, \delta\right) u_{i}=\exp \left(\delta^{\prime} z_{i t}\right) u_{i}, u_{i} \geq 0
$$

Some appealing features of this model are worth highlighting. First, due to the presence of $u_{i}$, inefficiency is correlated over time, i.e. $\operatorname{cov}\left(u_{i t}, u_{i t-1}\right) \neq 0$. This feature allows the inefficiency level to be estimated consistently when $T \rightarrow \infty$. In addition, since the inefficiency term is modelled as the product of a deterministic function of exogenous variables and a stochastic term, the marginal effect on inefficiency of a change in $z$ is separable into a deterministic and a stochastic component:

$$
\frac{\partial E\left(u_{i t}\right)}{\partial z_{i t}}=\frac{\partial g\left(z_{i t}, \delta\right)}{\partial z_{i t}} E\left(u_{i}\right)
$$

\section{Estimation issues}

In this section we will concentrate on a crucial topic in the identification of efficiency, namely controlling for other sources of unobserved heterogeneity. Inefficiency is an unobserved effect that explains differences in performance across firms. However, two other broad categories of unobserved heterogeneity can hamper the identification of inefficiency, namely, technical change and differences of technology across firms.

\subsection{Separating technical efficiency from technical change}

When panel data are available, the typical production frontier accounts for both inefficiency and technical change. However, Kumbhakar and Lovell (2000; p. 107) have recognized that "it may be difficult to disentangle the separate effects of technical change and technical efficiency change when both effects are proxied by the passage of time". Alvarez and del Corral (2006) performed Monte Carlo simulations in order to study the ability of several stochastic frontier models to correctly identify these two effects. Their results show that when both technical change and efficiency change are present in the data generating process most models fail to correctly distinguish between the two effects.

\subsection{Controlling for technological differences}

Some units are inherently very different in many aspects that are not reflected in the sample data and that can be interpreted as "technological" differences. It is desirable to distinguish between the inefficiency term and these components of 'unobserved heterogeneity'.

\subsubsection{Stochastic frontier with fixed effects}

When these characteristics have very little or no temporal variability and can therefore be assumed 'time-invariant', the model to be estimated can be written as:

$$
y_{i t}=\alpha_{i}+\beta x_{i t}+v_{i t}-u_{i t}
$$

Equation (7) differs from the usual stochastic frontier because it combines the individual fixed effects $\alpha_{i}$ with a composed error specification. This model, which was first suggested by 
Kumbhakar and Hjalmarsson (1993), has not been applied much in the empirical literature, most likely because the estimation by generalized least squares in its original formulation was complicated. Greene (2002) developed a maximum likelihood estimator which greatly simplifies its estimation and has generated a wave of empirical applications. ${ }^{3}$

\subsubsection{Latent Class Models and Inefficiency}

In recent years several alternatives have been proposed to relax the restrictive assumption that all firms share the same technological parameters. Tsionas (2002) and Greene (2005) have developed different versions of random coefficient models in which cross-firm heterogeneity is modelled in the form of continuous parameter variation. An alternative approach is to use Latent Class Models (LCM) to estimate the number of groups in the sample as well as the technology of each group. Following Orea and Kumbhakar (2004) we can write equation (1) as a latent class model as follows:

$$
y_{i t}=\left.f\left(x_{i t}\right)\right|_{j}+\left.v_{i t}\right|_{j}-\left.u_{i t}\right|_{j}
$$

where the vertical bar means that there is a different model for each class $j$. In fact, a different model implies different parameters to be estimated. A key aspect of LCM is the need to estimate 'a priori' probabilities of class membership in order to identify the different technologies and also as a way to assign firms to each group.

A subtle feature of the $L C M$ for panel data is that prior probabilities are modelled as time invariant ${ }^{4}$. In practical terms, this assumption implies that firms cannot switch between technologies over time. This seems to be rather restrictive since one would expect some firms to move across groups over the sample period. Alvarez and Arias (2013) propose a solution to this problem by estimating a LCM model in a way that allows firms to change technology over the period of analysis.

An interesting issue is that in a LCM firm inefficiency can be measured with respect to all frontiers. In practice, it is common to measure efficiency with respect to the most likely frontier (the one with the highest posterior probability), although it is possible to use a weighted average of the technical efficiencies for all the frontiers, using the posterior probabilities as weights.

At this point it is important to note that in the context of a LCM the finding that a group has higher (average) efficiency means that firms in that group are closer to their own frontier. However, it could be the case that the frontier of a group with high efficiency is located below the frontier of another group with lower efficiency. Therefore, in a LCM we are not only interested in finding which group of firms use the technology more efficiently but also in knowing which technology is more efficient. In order to compare the relative position of technologies, Alvarez and del Corral (2010) considered that a technology is locally above the others if its average predicted output is larger than that of other technologies. Obviously it may be the case that two frontiers cross. In this case, the mean of predicted output is not sufficient to describe the relative position of the frontiers and other summary information, such as the number of firms in the group with higher frontier output, could be used.

\footnotetext{
${ }_{4}^{3}$ See, for example, Alvarez (2007).

4 In a LCM the a priori probabilities of class membership can be specified as functions of some observed 'discriminant' variables in order to sharpen the estimation of these probabilities. Some of these variables can be time-varying. If this is the case, an econometric package like Limdep (see Greene, 2007) uses the individual mean of the time-varying variables in order to keep the time-invariance of the a priori probabilities.
} 


\section{Conclusions}

In this short note we have revisited several issues that are important in order to properly model stochastic frontiers. The models reviewed in the present paper deal rigorously with both difficult and relevant issues. No doubt these models can be improved or extended in the near future. However, a word of caution comes to mind after two decades of experience. First, very often the models aim to identify tenuous and overlapping effects. Therefore, we are not optimistic about increasing the complexity of the empirical models if there is not a similar effort in gathering good data. Second, some estimation techniques can come to the rescue when dealing with complicated models (e.g. Bayesian...). However, there is a risk of losing sight of the basic aims of this literature due to estimation complexity.

\section{References}

Ahn, S.C., Lee, Y.H. and Schmidt, P. (2007) Stochastic frontier models with multiple time-varying individual effects, Journal of Productivity Analysis, 27(1), 1-12.

Aigner, D., Lovell, K. and Schmidt, P. (1977) Formulation and estimation of stochastic frontier production function models, Journal of Econometrics, 6, 21-37.

Alvarez, A. (2007) Decomposing regional productivity growth using an aggregate production frontier, Annals of Regional Science, 41, 431-441.

Alvarez, A., Amsler, C., Orea, L. and Schmidt, P. (2006) Testing the scaling property in models where inefficiency depends on firm characteristics, Journal of Productivity Analysis, 25(3), 201-212.

Alvarez, A. and Arias, C. (2003) Diseconomies of size with fixed managerial ability in dairy farms, American Journal of Agricultural Economics, 85(1), 136-144.

Alvarez, A. and Arias, C. (2004) Technical efficiency and size. A conditional analysis, Agricultural Economics, 30(3), 241-250.

Alvarez, A. and Arias, C. (2013) Are dairy farms becoming more intensive? Impact on farm efficiency, Efficiency Series Paper 1/2013, Department of Economics, University of Oviedo.

Alvarez, A., Arias. C. and Greene, W. (2005) Accounting for unobservables in production models: management and inefficiency, Efficiency Series Paper 7/2005, Department of Economics, University of Oviedo.

Alvarez, A., Arias, C. and Kumbhakar, S. (2003) Empirical consequences of direction choice in technical efficiency analysis, Efficiency Series Paper 2/2003, Department of Economics, University of Oviedo.

Alvarez, A. and del Corral, J. (2006) Separating catch-up and technical change in stochastic frontier models: a Monte Carlo approach, Efficiency Series Paper 3/2006, Department of Economics, University of Oviedo.

Alvarez, A. and del Corral, J. (2010) Identifying different technologies using a latent class model. Intensive vs. extensive dairy farms, European Review of Agricultural Economics, 37(2), 231250.

Alvarez, A. and Schmidt, P. (2006) Is skill more important than luck in explaining fish catches?, Journal of Productivity Analysis, 26(1), 15-25.

Battese, G.E. and Coelli, T. (1992) Frontier production functions, technical efficiency and panel data: with application to paddy farmers in India, Journal of Productivity Analysis, 3, 153-169.

Battese, G.E. and Coelli, T. (1995) A model for technical efficiency effects in a stochastic frontier production function for panel data, Empirical Economics, 20, 325-332.

Cornwell, C., Schmidt, P. and Sickles, R. (1990) Production frontiers with cross-sectional and timeseries variation in efficiency levels, Journal of Econometrics, 46, 185-200.

Farrell, M.J. (1957) The measurement of production efficiency, Journal of the Royal Statistics Society A, 120, 253-281.

Gonzalez, E. and Alvarez, A. (2001) From efficiency measurement to efficiency improvement, European Journal of Operational Research, 133(3), 58-66.

Greene, W. (2002) Alternative panel data estimators for stochastic frontier models, mimeo, Stern School of Business, New York University. 
Greene, W. (2005) Reconsidering heterogeneity in panel data estimators of the stochastic frontier model, Journal of Econometrics, 126(2), 269-303.

Greene, W. (2007) Limdep user's guide v. 9.0, Econometric Software.

Huang, C.J. and Liu, J.T. (1994) Estimation of a non-neutral stochastic frontier cost function, Journal of Productivity Analysis, 5, 171-180.

Kopp, R.J. (1981) The measurement of productive efficiency. A reconsideration, The Quarterly Journal of Economics, 96(3), 477-503.

Kumbhakar, S. (1990) Production frontiers, panel data and time-varying technical inefficiency, Journal of Econometrics, 46, 201-211.

Kumbhakar, S.C. and Hjalmarsson, L. (1993) Technical efficiency and technical progress in Swedish dairy farms, in Fried, H., Lovell, C.A.K. and Schmidt, S.S. (eds.) The Measurement of Productive Efficiency: Techniques and Applications, Oxford University Press: New York.

Kumbhakar, S., Ghosh, S. and McGuckin, J.T. (1991) A generalized production frontier approach for estimating determinants of inefficiency in U.S. dairy farms, Journal of Business and Economic Statistics, 9, 279-286.

Kumbhakar, S.C. and Lovell, C.A.K. (2000) Stochastic Frontier Analysis, Cambridge University Press.

Lee, Y.H. and Schmidt, P. (1993) A production frontier model with flexible temporal variation in technical efficiency, in Fried, H., Lovell, C.A.K. and Schmidt, S.S. (eds.) The Measurement of Productive Efficiency: Techniques and Applications, Oxford University Press: New York.

Meeusen, W. and van den Broeck, J. (1977) Efficiency estimation from Cobb-Douglas production functions with composed error, International Economic Review, 18(2), 435-444.

Mundlak, Y. (1961) Empirical production functions free of management bias, Journal of Farm Economics, 43, 44-56.

Orea, L., Roibás, D. and Wall, A. (2004) Choosing the technical efficiency orientation to analyze firms' technology. A model selection test approach, Journal of Productivity Analysis, 22(1), $51-71$.

Orea, L. and Kumbhakar, S. (2004) Efficiency measurement using a stochastic frontier latent class model, Empirical Economics, 29, 169-183.

Reifschneider, D. and Stevenson, R. (1991) Systematic departures from the frontier: a framework for the analysis of firm inefficiency, International Economic Review, 32, 715-723.

Reinhard, S., Lovell, C.A.K. and Thijssen, G. (1999) Econometric estimation of technical and environmental efficiency: an application to Dutch dairy farms, American Journal of Agricultural Economics, 81(1), 44-60.

Schmidt, P. and Sickles, R. (1984) Production frontier and panel data, Journal of Business and Economics Statistics, 2, 367-374.

Tsionas, E.G. (2002) Stochastic frontier models with random coefficients, Journal of Applied Econometrics, 17, 127-147.

Wang, H.J. (2002) Heteroscedasticity and non-monotonic efficiency effects of a stochastic frontier model, Journal of Productivity Analysis, 18, 241-253.

Wang, H.J. and Schmidt, P. (2002) One-step and two-step estimation of the effects of exogenous variables on technical efficiency levels, Journal of Productivity Analysis, 8, 129-144. 